\title{
Irrigation network design and reconstruction and its analysis by simulation model
}

\author{
Milan Čistý, Zbynek Bajtek, Anna Becová \\ Slovak University of Technology in Bratislava \\ Faculty of Civil Engineering, Department of Land and Water Resources Management \\ e-mail:milan.cisty@stuba.sk, zbynek.bajtek@stuba.sk, anna.becova@stuba.sk
}

\begin{abstract}
There are many problems related to pipe network rehabilitation, the main one being how to provide an increase in the hydraulic capacity of a system. Because of its complexity the conventional optimizations techniques are poorly suited for solving this task. In recent years some successful attempts to apply modern heuristic methods to this problem have been published. The main part of the paper deals with applying such technique, namely the harmony search methodology, to network rehabilitation optimization considering both technical and economic aspects of the problem. A case study of the sprinkler irrigation system is presented in detail. Two alternatives of the rehabilitation design are compared. The modified linear programming method is used first with new diameters proposed in the existing network so it could satisfy the increased demand conditions with the unchanged topology. This solution is contrasted to the looped one obtained using a harmony search algorithm.
\end{abstract}

Key words: irrigation networks, network rehabilitation, harmony search

\section{Introduction}

Some of the larger sprinkler irrigation systems, which were built in the Slovak Republic starting from early fifties [1], are now approaching the end of their useful life. Some of them are out of operation, due to their wear-out, obsoleteness or more serious failures. The private ownership of land became more diverse. Hence now, in the majority of irrigation networks, there are more consumers of irrigation water. As a result, there is higher demand for irrigation water during the peak period than is available in the system due to its limited capacity, i.e. both irrigation piping system and pump stations. That is why rehabilitation and modernization of these systems has become a significant interest for engineers today. Modernization and rehabilitation is becoming more important also in the field of water distribution of drinking water [2].

In the majority of cases the requirement to increase the hydraulic capacity of the system is based on the following demands: 
- To increase pressure and take off at demand points due to the irrigators upgrade (with increased demand).

- To provide sufficient pressure within the pipeline system with a larger number and modified locations of demand points as well as their grouping in selected parts of an irrigation system.

- To expand the system by adding new branches.

- To eliminate system deficiencies due to its aging.

In the majority of these cases it is not feasible to increase pressure in the pump station since the pressure characteristics of the pipe material do not allow it. The increase in the hydraulic capacity of the system is thus possible only by changing the pipe network. The purpose of this paper is to explore the use of linear programming and harmony search algorithm optimization techniques to the design and rehabilitation of pipeline irrigation systems.

The linear programming (LP) method has long been accepted as an approach for the optimal selection of diameters for pipes in branched networks. Mathematical models based on LP were developed and used in the irrigation network design process [6]. However, problems with adapting mathematical programming methods to looped network problems have restricted the use of LP to branched layouts only. Majority of pressurized irrigation systems with large area coverage was designed with a branched layout. At first glance this seems to be only negative aspect because from both hydraulic and operational viewpoint looped networks are usually preferred to branch networks. The main disadvantage of branched networks is that they provide only one route to the delivery point. Paradoxically, networks without loops in the original configuration may be viewed as an advantage since they may be improved by introducing loops. It represents a precious potential of branched networks, especially in the case when there is need to increase their hydraulic capacity as a part of rehabilitation of irrigation networks.

This option is even more valuable when we consider the fact that in the beginning of previous decade several papers appeared in the scientific literature, presenting modern heuristics capable of providing solutions to optimization problems where previously deterministic algorithms have failed. One of the heuristic methods, which are suited for purposes of design, rehabilitation or calibration of pressurized pipe networks, is the harmony search algorithm (HS). Since it is not the purpose of this article to analyses in detail possibilities, techniques and developmental trends of heuristic methods in general, authors will stick to the basic description of applied algorithm. Both algorithms has been integrated with EPANET hydraulic network solver [4] in Visual Basic.

\section{Methods}

\subsection{Harmony search algorithm}

The harmony search algorithm [3] was conceptualized from the musical process of searching for a 'perfect state' of harmony, such as jazz improvisation. Jazz improvisation seeks a best state (best harmony) determined by aesthetic estimation, just as the optimization algorithm seeks a best state (global optimum) determined by evaluating the objective function. Aesthetic estimation is performed by the set of notes played by each instrument, just as the objective function evaluation is performed by the set of values assigned by each decision variable. The 
harmony quality is enhanced by practice, just as the solution quality is enhanced iteration by iteration.

The steps in the harmony search algorithm are as follows:

Step 1. Initialize the problem and HS parameters, these parameters are the harmony memory size (HMS), harmony memory considering rate (HMCR), pitch adjusting rate (PAR) and the number of improvisations (NI).

Step 2. Initialize the harmony memory, in this step the HM matrix is filled with as many randomly generated solution vectors as the HMS.

Step 3. Improvise a new harmony, generating a new harmony.

The procedure is shown in a pseudo code:

for each iv $[1, N]$ do

if $U(0,1) \leq H M C R$ then 'memory consideration

begin

$x_{i}=x_{j i}, \quad$ where $j \sim U(1, \ldots, H M S)$.

if $U(0,1) \leq P A R$ then 'pitch adjustment

begin

$x_{i}=x_{i} \pm r x b w$, where $r \sim U(0,1)$

endif

else' random selection

$x_{j}=L B_{i}+r x\left(U B_{i}-L B_{i}\right)$

endif

\section{Done}

Step 4 Update harmony memory, If the new harmony vector is better than the worst harmony vector in the HM, judged in terms of the objective function value, and no identical harmony vector is stored in the HM, the new harmony is included in the HM and the existing worst harmony is excluded from the HM.

Step 5 Check the stopping criterion, Steps 3 and 4 are repeated until the termination criterion is reached.

Meaning of symbols is as follows. HMS - the harmony memory size; generally varies from 1 to 100 (typical value $=30$ ). HMCR - the rate of choosing a value from the harmony memory; generally varies from 0.7 to 0.99 (typical value $=0.9$ ). PAR - the rate of choosing a neighbouring value. Value of these parameters depends on the type of optimization problem.

\subsection{Optimization of the Branched Network Using Linear Programming}

For the clarity purposes we briefly describe the optimization procedure of the pipe network rehabilitation using linear programming. The mathematical formulation of this problem is as follows:

$$
\begin{aligned}
& A_{11} x_{1}+A_{12} x_{2}+\ldots+A_{1 n} x_{n}=B_{1} \\
& A_{21} x_{1}+A_{22} x_{2}+\ldots+A_{2 n} x_{n}=B_{2}
\end{aligned}
$$

etc. 


$$
\begin{gathered}
A_{m 1} x_{1}+A_{m 2} x_{2}+\ldots+A_{m n} x_{n}=B_{m} \\
c_{1} x_{1}+c_{2} x_{2}+\ldots+c_{m} x_{n}=\min (\max )
\end{gathered}
$$

Solution has to comply with inequalities:

$$
x_{1}>\mathbf{0} ; x_{2}>\mathbf{0} \text { etc. up to } x_{n}>\mathbf{0}
$$

When in order to resolve pipeline networks rehabilitation task we apply linear programming, unknown will be the lengths of individual pipeline diameters. In first conditions we must mathematically express the requirement that the sum of unknown lengths of individual diameters in each section has to be equal to its total length. The second type of the equation will represents the request that the total pressure losses in a hydraulic path between the pump station and critical node (the end of the pipeline, extreme elevation inside the network) should be equal or less than the known value. This constraint is based on the maximum network pressure requirement needed for the operation of the system. Given the investment costs minimization requirement, the objective function sums the products of individual pipeline prices and their required lengths. When we formulate the rehabilitation optimization, the objective function should provide economical advantage for the choice of original diameters; however it should not discriminate against the choice of a larger diameter (if the hydraulic situation requires so). Four possible diameters are selected for each section. The first possible option is the diameter that is identical with the original one in that section, with the three larger diameters as options for the same section. In the objective function the program assigns the original diameter a minimum unit price and real prices to other diameters. Further details on LP optimization can be found in available literature [6].

\section{Results and discussion}

The described methods of the optimal pipe network rehabilitation were applied to the irrigation system Tomášovo. Its layout is shown in the Figure 1. This is one of the irrigation facilities with large area coverage in Slovakia, with applied sprinkler irrigation and an underground pressurized water network. Its construction was completed at the beginning of the sixties and thus the whole facility is coming close to the end of its service life and hence it can serve as a suitable model for testing the proposed rehabilitation methods.

The irrigated area amounts to 700 ha. The irrigation system consists of irrigation water take off complex located at the irrigation inlet to the irrigation pump station, pump station itself, pressurized network for the delivery of irrigation water and sprinklers. Only the basic parameters (pressure and output of the pump station, required pressure and sprinklers take off) are taken into account. Four loops were added to the network (between nodes 20-87, 91-148, 155- 166 and 182-187).

The design of the system was substantially based upon the concept of hand-move laterals. Since this approach is now abandoned, its use is not producing required benefits. This is the reason why we have decided to review the rehabilitation proposals based on the concept of irrigation with non-specific hose-reel irrigators with an optimum output of $7 \mathrm{ls}^{-1}$ and optimal inlet pressure $0,4-0,7 \mathrm{MPa}(0,55-0,60 \mathrm{MPa}$ was used as allowed minimal pressure in computations). In addition to that it is assumed that a battery of such sprinklers will be used, 
i.e. there will be four variants of serial set of three, four, five and six machines. The original network is unable to comply with hydraulic requirements for such operations. The configuration of irrigators could be chosen arbitrarily without any impact on the calculation procedure presented in this paper.

The procedure could be summarized as follows:

1. The mathematical model of the branched network was first set up for rehabilitation optimization using linear programming.

2. The mathematical model of the looped network was then set up for rehabilitation optimization using HS.

3. Economical and technical comparison of the branched and looped rehabilitation alternatives was performed by simulating high number of possible demand situations (placement of irrigators).

\subsection{Optimisation of the Network Using Harmony search algorithm}

The following set of HS parameters was used for optimising the looped network design. Harmony memory size (HMS) was set to 35, harmony memory considering rate (HMCR) at 0.95 and pitch adjusting rate (PAR) to value 0.05.The HS was allowed to run until full convergence, i.e. until all members of the final population are equal. It took HS between 250,000 and 350,000 iterations to arrive at the best design. This indicates that the optimum is more difficult to find in the case of looped network.

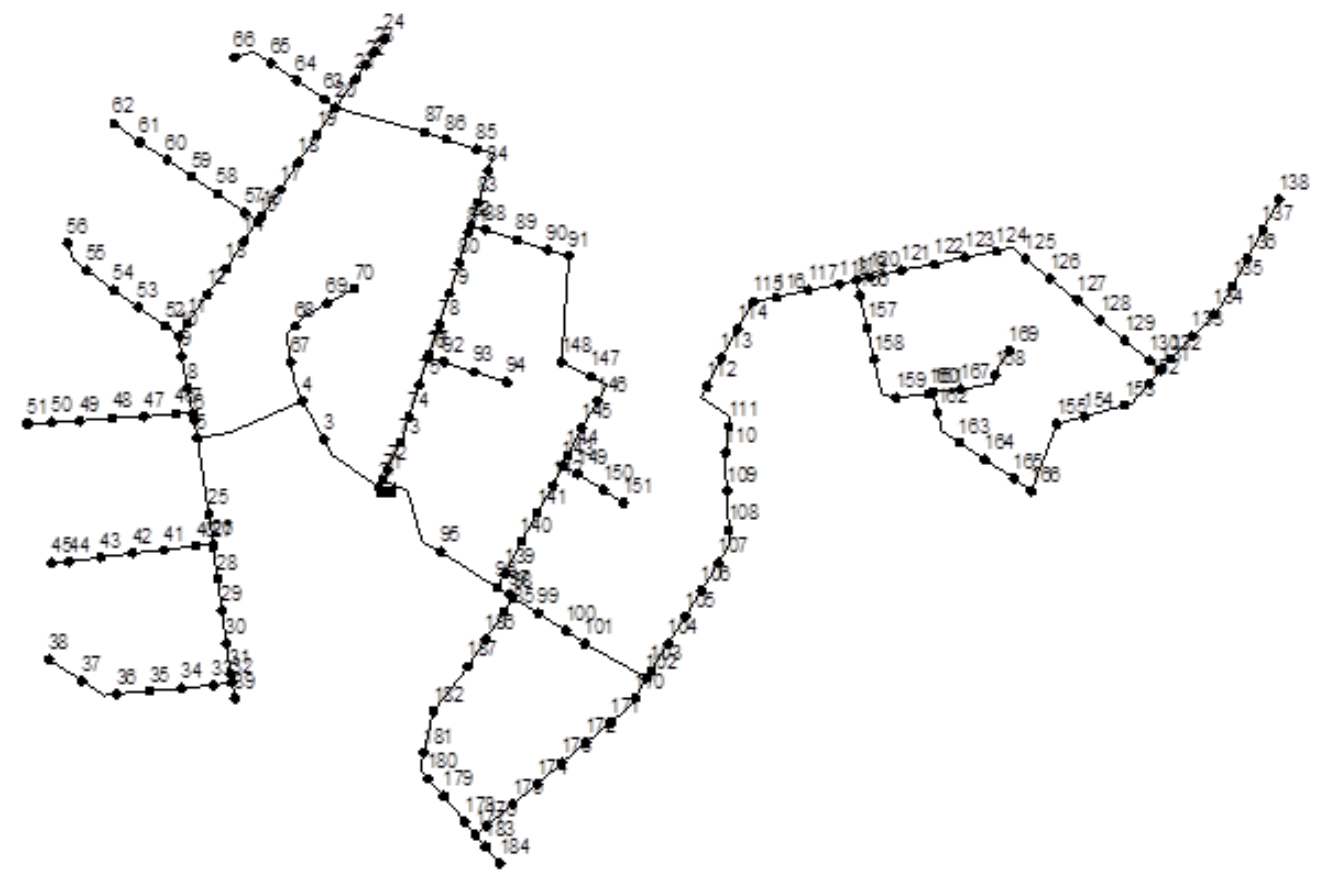

Figure 1: Diagram of the looped network with node numbers (HS) 
Table 1: Cost of the network when harmony search is been used

\begin{tabular}{|c|c|c|c|}
\hline \multicolumn{4}{|c|}{ Harmony search } \\
\hline Pipe diameter & Pipe length & Cost & Sum \\
\hline$[\mathrm{mm}]$ & {$[\mathrm{m}]$} & {$[$ Euro/m] } & v Euro \\
\hline 500 & 84,02 & 72,5 & 6091,45 \\
\hline 350 & 2186,95 & 47 & 102786,7 \\
\hline 300 & 3162,23 & 36,5 & 115421,4 \\
\hline 225 & 2403,28 & 25 & 60082 \\
\hline 200 & 6248,33 & 20 & 124966,6 \\
\hline 150 & 6939,42 & 15,5 & 107561 \\
\hline 100 & 4452,26 & 12 & 53427,12 \\
\hline
\end{tabular}

\subsection{The Comparison of Branched and Looped Rehabilitation Solutions}

The obtained results indicate that the calculation using linear programming provides from economic point of view some better results than calculation using HS on looped network. This follows from the fact, that LP has a deterministic algorithm, which provides under the definition of the solution in the way, it has been formulated in the mathematical model real global minimum. Furthermore, in looped version of the network are more pipes than in branched one. This is completely new situation different from the one using deterministic algorithms (e.g. non-linear programming), where the occurrence of loops within the network makes the task very difficult to be resolved. Beside branched alternative acquired by LP we also create a looped alternative from it (Figure 2), where we introduce new connection pipes. This step as will be explained later should help increase hydraulic stability of the network.

Table 2: Cost of the network when linear programing is been used

\begin{tabular}{|c|c|c|c|c|c|c|}
\hline \multicolumn{4}{|c|}{ Linear Programming branch network } & \multicolumn{3}{|c|}{ Linear Programming looped network } \\
\hline Pipe diameter & Pipe length & Cost & Sum & Pipe length & Cost & Sum \\
\hline$[\mathrm{mm}])$ & $([\mathrm{m}])$ & [Euro/m] & v Euro & $[\mathrm{m}])$ & {$[$ Euro/m] } & v Euro \\
\hline 500 & 69,3 & 72,5 & 5024,25 & 69,3 & 72,5 & 5024,25 \\
\hline 350 & 2387,4 & 47 & 112207,8 & 2194,78 & 47 & 103154,7 \\
\hline 300 & 2906,6 & 36,5 & 106090,9 & 3096,21 & 36,5 & 113011,7 \\
\hline 225 & 1246,8 & 25 & 31170 & 2157,1 & 25 & 53927,5 \\
\hline 200 & 7502,6 & 20 & 150052 & 7922,91 & 20 & 158458,2 \\
\hline 150 & 6515,9 & 15,5 & 100996,5 & 6994,56 & 15,5 & 108415,7 \\
\hline 100 & 3426,3 & 12 & 41115,6 & 4535,1 & 12 & 54421,2 \\
\hline
\end{tabular}




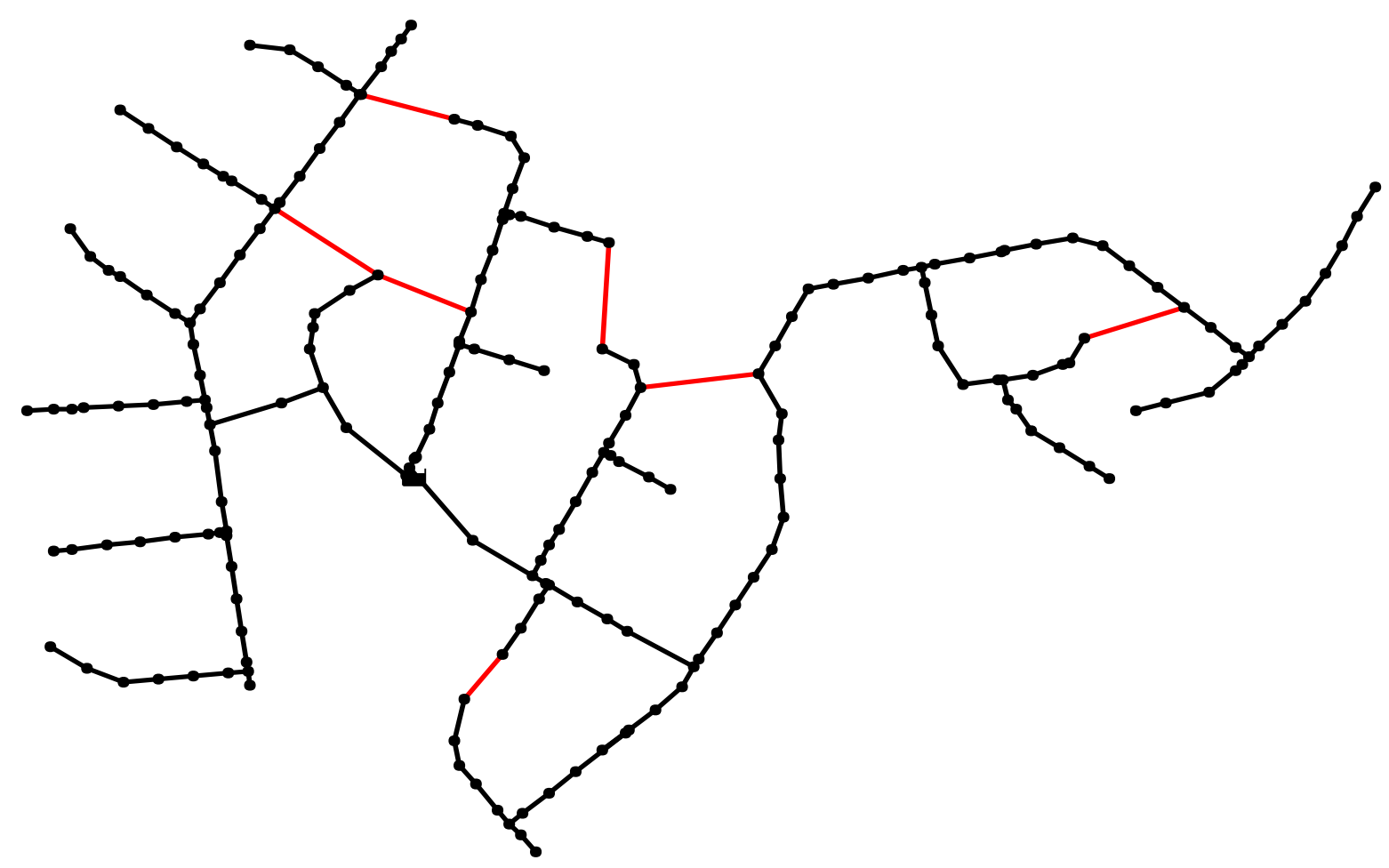

Figure 2: Diagram of the network from LP design with additional pipes

\subsection{Pressure assessment in simulation model}

The pressure assessment of the pipeline network was done in such a way that a set of realistic demand situations with maximum hydraulic requirements (compatible with that ones used for design of the network) is analysed. These demand situations differ by placement of irrigators in irrigated area and also the gap between irrigators groups. We have tested 4 alternatives of group sizes (3,4,5,6 irrigators in one group) and tree possible alternatives of omission of demand nodes $(8,12,16$ omitted nodes). In these alternatives we have assessed the minimum and average pressures at all demand points. These values are shown in diagram (Figure 3,4), where the data is sorted according to value (Pressure). For better illustration we present in the table 3 number of demand situation when the pressure in the tested network is under 0.45 $[\mathrm{MPa}]$. This value represents minimum pressure required by the irrigators to operate normally.

The procedure to obtain these results was as follows:

- A plan of the realistic critical demands and their locations was prepared (for all configurations). The critical locations of the irrigation groups are those which cases higher maximum pressure requirements within the system.

- Since the real number of simultaneously operating irrigator groups in a single operational situation is smaller than their potential number, it was necessary to define the number of irrigators and some other parameters for tested demand situations. According this conditions the structure of each demand scenario was also determined (i.e. the demand locations in each of them). This is de facto combinatorial task. 
- The final step was to run simulation calculation for all these operational situations on the branched and looped networks configurations.

Simulation results prove the benefit of looping both in hydraulic terms (better pressure ratios) and in economic terms - creating loops in network is cheaper opinion for rehabilitation than LP solution acquired by redesigning the network (this applies only when creating loops by applying new connection pipes is only intervention to the network). One can assume that the described result is applicable also for other systems. This is due to the fact, that both in Slovakia as well as in the rest of the world the trend was to design branched pipe networks for irrigation.

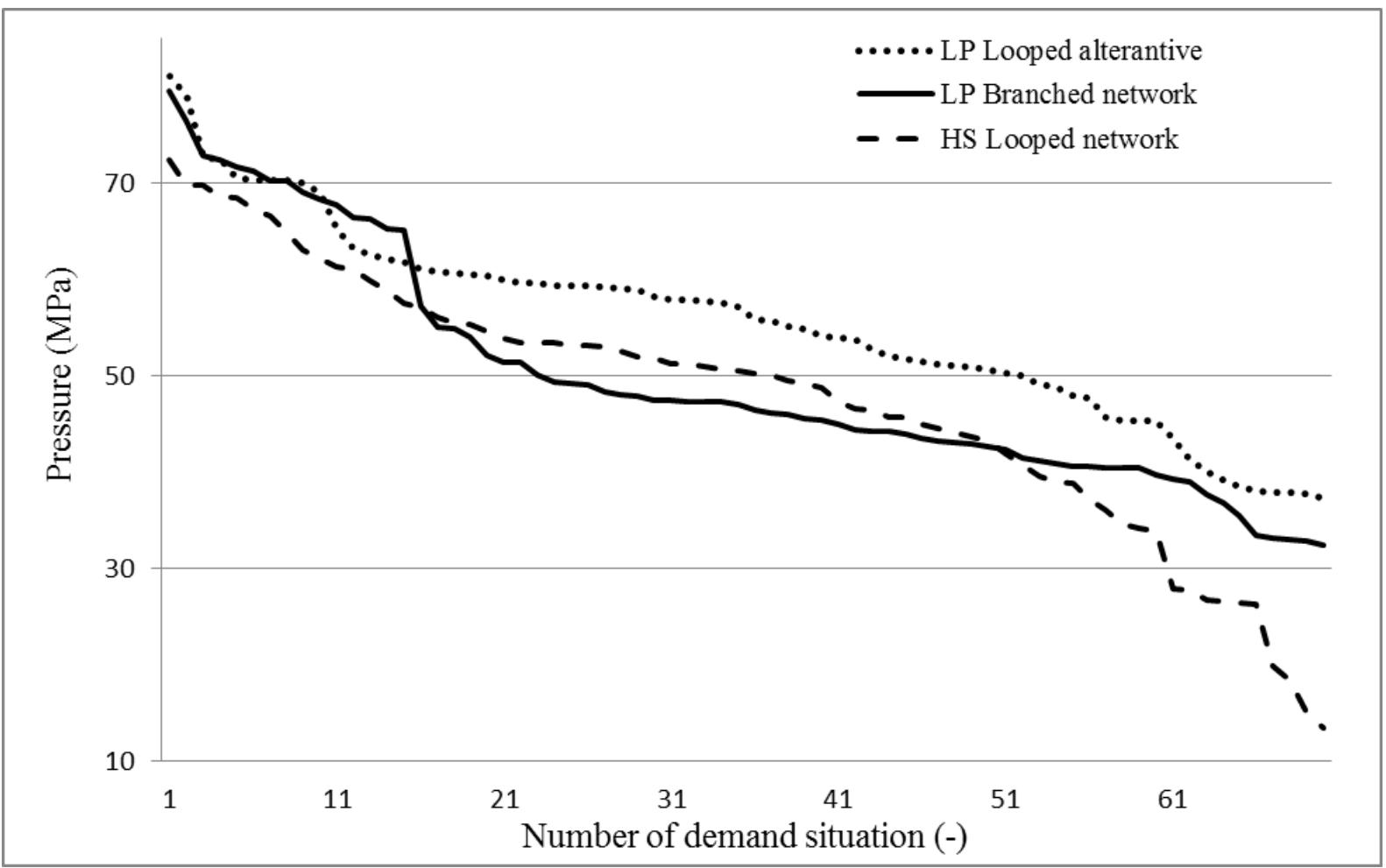

Figure 3: Lined up minimal pressures in networks (five irrigators)

Economical and hydraulic effectiveness of the rehabilitation of the sprinkler irrigation systems highly depend on the effectiveness of their pipe network rehabilitation. Irrigation pipe networks are often branched and an obvious approach to increase the hydraulic capacity of such systems while keeping capital investment at minimum is to convert these systems to looped networks. Because of their complexity and inability to cope well with discrete, nonlinear combinatorial problem, such as pipe network optimization, the conventional optimization techniques are poorly suited for solving this task.

This was again confirmed in this study by applying a classical optimization methodology, linear programming, and the harmony search algorithm (HS) to network rehabilitation. 
Table 3: Pressure analysis of different demand scenarios

\begin{tabular}{|c|c|c|c|c|c|c|c|c|c|c|c|c|c|}
\hline \multirow{2}{*}{\multicolumn{2}{|c|}{$\begin{array}{l}\text { Number of irrigators in group } \\
\text { Gap between each group(number of nodes } \\
\text { without demand) }\end{array}$}} & \multicolumn{3}{|c|}{3} & \multicolumn{3}{|c|}{4} & \multicolumn{3}{|c|}{5} & \multicolumn{3}{|c|}{6} \\
\hline & & 8 & 12 & 16 & 8 & 12 & 16 & 8 & 12 & 16 & 8 & 12 & 16 \\
\hline \multicolumn{2}{|c|}{$\begin{array}{l}\text { Total number of evaluated demand } \\
\text { situation }\end{array}$} & 75 & 66 & 66 & 80 & 72 & 68 & 75 & 70 & 60 & 78 & 72 & 66 \\
\hline \multirow{3}{*}{$\begin{array}{l}\text { Number of } \\
\text { situations with } \\
\text { pressure under } \\
0.45[\mathrm{Mpa}]\end{array}$} & LP - Branch network & 33 & 20 & 17 & 32 & 28 & 51 & 37 & 30 & 59 & 52 & 42 & 53 \\
\hline & \begin{tabular}{|l|} 
LP - Looped \\
alternative
\end{tabular} & 36 & 15 & 12 & 24 & 23 & 19 & 18 & 10 & 9 & 29 & 25 & 22 \\
\hline & HS - Looped network & 23 & 18 & 15 & 30 & 24 & 20 & 35 & 13 & 8 & 30 & 26 & 22 \\
\hline
\end{tabular}

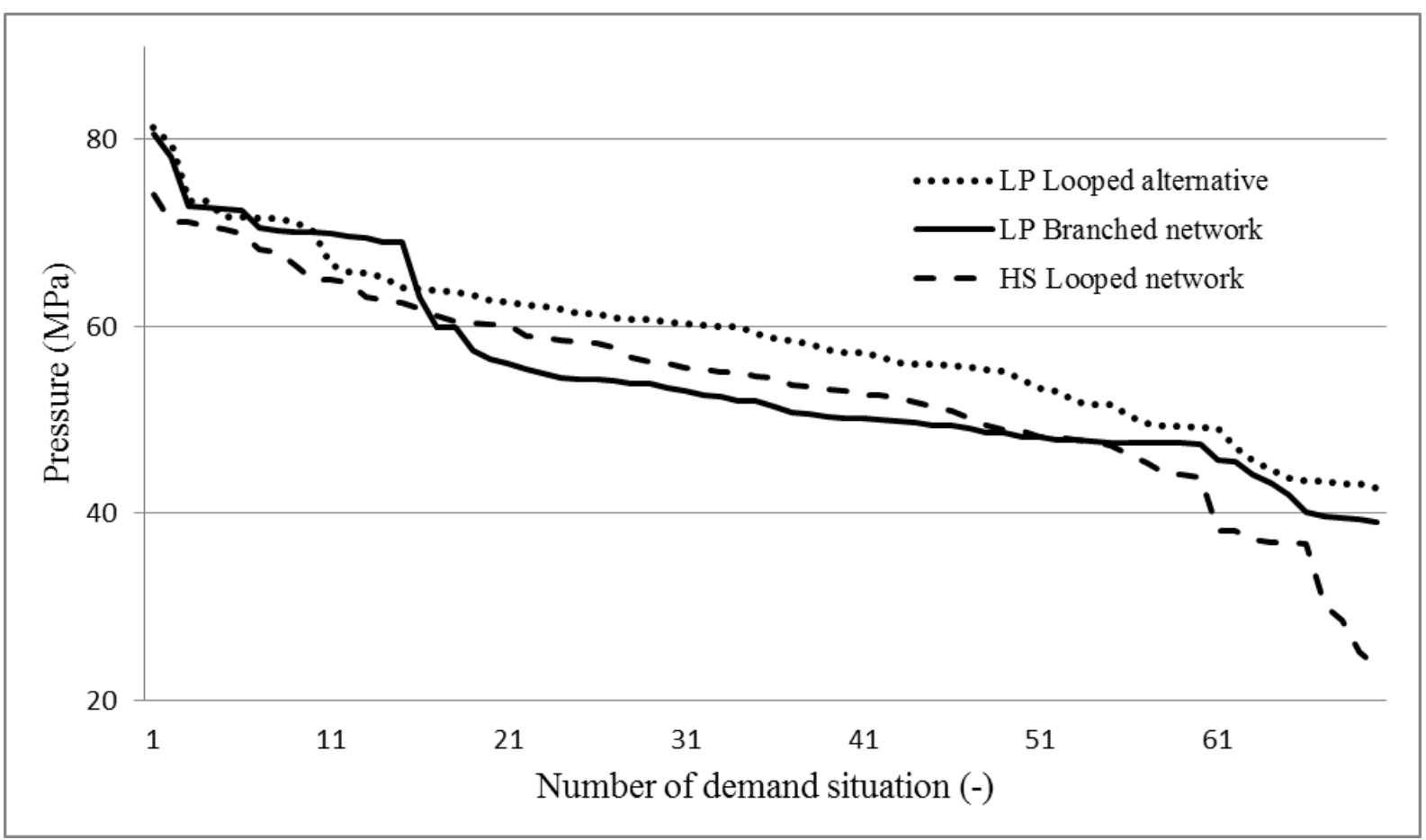

Figure 4: Lined up average pressures in network (five irrigators)

\section{Conclusion}

Main reason for this article was to point out advantages and disadvantages of different irrigation network designs. We used two approaches to design of irrigation network one using standard deterministic method (LP) to design branched network and second heuristic method (HS) to design looped alternative. Furthermore we try to improve hydraulic stability of the branched network designed by LP by adding additional pipes to create a looped alternative from it. In the second part we have evaluated these networks for different demand scenarios not only to determine optimal operation of the irrigation network (Table 3) but also to show 
benefits of introducing loops to a branch network. Here we can see combination of irrigation scenarios for 3,4,5,6 irrigators and three sizes of gap between each group. These parameters define total number of evaluated demand situations. The last three rows represent the total number of demand situation when the pressure requirements where not satisfied. For testing we have used a simulation models which evaluated network for various demand condition. Results proofed that by using LP we can obtain lower cost for the network design but on the other hand the network is less stable (by the means of required minimum pressure) then looped network. Advantage of the low cost of the network from LP is at the expense of the stability of the network from hydraulic point of view. This disadvantage we try to overcome by introducing new pipes to the branched network optimized by LP creating alternative to the looped network optimized by HS and originally branched network from LP. This step proof to by useful as the output from simulation models show (Figure 3,4). We can see that this alternative outperform both other alternatives (branched network from LP and also looped network from HS) by all means. It's even more important when we realize that most of irrigation networks were originally designed as a branched network, so the introducing of loops could improve system operation in changing environment.

\section{Acknowledgements}

This work was supported by the Scientific Grant Agency of the Ministry of Education of the Slovak Republic and the Slovak Academy of Sciences, Grants No. 1/1044/11 and 1/9008/11.

\section{References}

[1] Bodnar, J. and Jansky L. (1989). Irrigation in Czechoslovakia. Irrigation News, UK Irrigation Association, 1989, No. 15, p. 53-59.

[2] Halhal, D., Walters, G., Ouazar, D., and Savic, D.A., (1997). "Water Network Rehabilitation with Structured Messy Genetic Algorithm." J. Water Resour. Plann. Manage., 123(3), 137-146.

[3] Geem, Z.W. (2006). Optimal Cost Design of Water Distribution Networks Using Harmony Search,” Engineering Optimization, Vol. 38, No. 3, pp. 259-280.

[4] Rossman, L. A.,(2000). EPANET User's Manual, U.S. Environmental Protection Agency.

[5] Walters, G.A., Savic, D.A., M.S. Morley, W. de Schaetzen and R.M. Atkinson, (1998). Calibration of Water Distribution Network Models Using Genetic Algorithms, In Hydraulic Engineering Software VII, Computational Mechanics Publications, pp. 131-140, 1998.

[6] Zdražil K., (1968). Research of the computation and graphical methods of designing the irrigation pipe networks. VUZH Bratislava. In Czech. 